\title{
OBSESSIVE COMPULSIVE DISORDER AND ITS CARE-REVIEW
}

\author{
Shaik Aminabee*, Dasari Durga Jayashree and Atmakuri Lakshmana Rao \\ Department of Pharmacology, V. V. Institute of Pharmaceutical Sciences, \\ Gudlavalleru, Andhra Pradesh, India.
}

\begin{abstract}
Obsessive-compulsive disorder (OCD) features a pattern of unreasonable thoughts and fears (obsessions) that lead you to do repetitive behaviours (compulsions). These obsessions and compulsions interfere with daily activities and cause significant distress. You may try to ignore or stop your obsessions, but that only increases your distress and anxiety. Ultimately, you feel driven to perform compulsive acts to try to ease your stress. Despite efforts to ignore or get rid of bothersome thoughts or urges, they keep coming back. This leads to more ritualistic behaviour, the vicious cycle of OCD. OCD often centres on certain themes. To ease your contamination fears, you may compulsively wash your hands until they're sore and chapped. If you have OCD, you may be ashamed and embarrassed about the condition, but treatment can be effective.
\end{abstract}

Keywords: Obsessive compulsive disorder, Serotonin, Anti-depressant and Abnormal behaviour.

\section{INTRODUCTION}

If anyone who is constantly or repetitively involved in excessive, unreasonably behaviours like cleaning, hand washing or rearranging. Then that person is suffering from obsessive compulsive disorder (OCD). It is a psychiatric \& chronic disorder characterized by obsessive thoughts and compulsive actions, such as cleaning, checking, counting, or hoarding. Obsessive compulsive disorder (OCD), one of the anxiety disorders. OCD occurs in a small percentage of populations worldwide in every culture. The individual who suffers from OCD pattern behaviour senseless and distressing but extremely difficult to overcome (Jenike, 2004). Often the person carries out the behaviours to get rid of the obsessive thoughts. But this only provides short-term relief. Not doing the obsessive rituals can cause great anxiety and distress. OCD is related to anxiety and intrusive thoughts (obsessions) that often result in repetitive behaviour (compulsions). Well many of us do suffer from mild form of this where we realize that our behaviours are little abnormal or stupid but these behaviours can be controlled voluntarily. Obsessions are persistent and recurrent thoughts that cause emotional distress, such as disgust or anxiety (Lysaker et al., 2000). Many OCD sufferers realize that their actions are unreasonable, but are unable to gain control through logic or reasoning.

Examples of obsessions include: Fear of contamination or germs, an irrational need for symmetry or order and aggressive thoughts about oneself or others.

Compulsions are mental urges to repeat certain behaviours to reduce or prevent a feared situation. In the most severe cases, this ritualistic repetitive behaviour may fill the day, making it impossible to perform other routines (Kessler et al., 2005). Examples of compulsions include: Excessive hand washing or cleaning and arranging and rearranging objects in a specific way etc.

These behaviours generally are intended to ward off harm to the person with OCD or others. Some people with OCD have regimented rituals while others have rituals that are complex and changing. Performing rituals may give the person with OCD some relief from anxiety, but it is only temporary. The old belief that OCD was the result of life experiences has been weakened by the growing evidence that biological factors are a primary contributor to the disorder. The fact that OCD patients respond well to specific medications that affect the neurotransmitter serotonin suggests he disorder has a neurobiological basis. This OCD can also have seen in teen (Angst et al., 2005). Actually, the 
teens who are with the OCD are feeling so stressful life and they stop the relieving and enjoyment of life they cannot feel the rest if suppose they are relaxing also it is just a temporary relaxing. And it is just temporary relaxing. And it is difficult to find out the obsessive-compulsive disorder patients in teens because they can try hide their symptoms. Children with OCD do not always realize that their obsessions or compulsions are unreasonable. They might have tantrums when prevented from completing rituals. Also in contrast to adults, children and teenagers tend to develop physical complaints like tiredness, headaches, and stomach upset when afflicted with OCD (Ruscio et al., 2010).

\section{HISTORY}

The incidence of Obsessive-Compulsive Disorder (OCD) as it was once known, is a relatively common disorder and can be traced historically, cross-culturally and across a broad social spectrum and does not appear to restrict itself to any specific group of individuals. Of course, the name OCD did not come into being until the $20^{\text {th }}$ century, but prior to that earlier references to symptoms we would now call Obsessive-Compulsive Disorder were surprisingly called "Scrupulosity". The Oxford Don, Robert Burton, reported a case in his compendium, the Anatomy of Melancholy (1621) and in 1660, Jeremy Taylor, Bishop of Down and Connor, Ireland, was referring to obsession doubting when he wrote of "scruples". A scruple is trouble where the trouble is over, a doubt when doubts are resolved (Aardema et al., 2007). Modern concepts of OCD began to evolve in the nineteenth century, when Faculty Psychology, phrenology and Mesmerism were popular theories and when "neurosis" implied a neuropathological condition. Like us, psychiatrists then struggling to understand the mentally ill were influenced by currents coursing through philosophy, physiology, physics, chemistry and political thought. In his 1838 psychiatric textbook, Esquirol (17721840) described OCD as a form of monomania, or partial insanity. He fluctuated between attributing OCD to disordered intellect and disordered will. The imperfect success of these defenses gave rise to OCD symptoms: anxiety; preoccupation with dirt or germs or moral questions; and, fears of acting on unacceptable impulses. As the twenty-first century begins, advances in pharmacology, neuro anatomy, neurophysiology and learning theory have allowed us to reach a more therapeutically useful conceptualization of OCD.

\section{EPIDEMIOLOGY}

Medicinal writings and National Institute of Mental Health have described about $1 \%$ in India and 5-10\% worldwide suffers with OCD. Such numbers as high as $60-70 \%$ of population Statistics on how many people in the United States have OCD range from 1-2\% or more than 2 million adults. About one in 200 children and adolescents, or half a million minors, has OCD. Interestingly, OCD does not discriminate and can affect anyone regardless of race, gender or age (Ab Boileau B, 2011). How often this condition occurs and the symptoms involved are remarkably similar across cultures. While it often starts in childhood and adolescence, the average age of onset of the disorder is 19 years of age. OCD usually develops by 30 years of age, afflicting more males than females. But this $1 \%$ data belongs to those whose abnormal or stupid behaviours become recurrent and can't be controlled voluntarily.

\section{TYPES OF OCD}

Although there are different forms of OCD it is mainly categorized into 5 main types (Abramowitz et al., 2009).

1. Checking

2. Symmetry and Ordering

3. Ruminations / Intrusive Thoughts

4. Hoarding

5. Contamination / Mental Contamination

\section{Checking}

Checking is often carried out multiple times, sometimes hundreds of times and might last for an hour or even longer causing significant impact on the person's life, being late for school, work, social occasions and other appointments. Like checking door lock or window, checking alarm, checking gas stoves etc.

\section{Symmetry and Ordering}

Those affected will spend a lot of time trying to get the symmetry 'just right' and this time consuming checking can result in them being extremely late for work and appointments. They may also become mentally and physically drained if the compulsions take a considerable amount of time like neatness, picture, tinned cans, clothes, books and CD's spotless without blemish (Germana Moretti, 2008).

\section{Rumination / Intrusive thoughts}

Rumination is a term often used to describe all obsessional intrusive thoughts, and the definition of rumination perhaps helps encourage that belief "a deep or considered thought about something", but this is slightly 
misleading from an OCD cortex. Example, thoughts of causing violent or sexual harm to loved ones which don't involve specific immediate compulsions these are called Intrusive Thoughts, and sometimes mistakenly referred to as 'Pure O'.

\section{Hoarding}

Another obsession long considered to be part of 'OCD' is the inability to discard useless or worn out possessions, commonly referred to as 'hoarding.

\section{Contamination / Mental Contamination}

The fear of being dirty and contamination is the obsessional worry, often fear is that contamination might cause harm to one's self or a loved one. The common compulsions might be to wash or clean or avoid. Common contamination obsessive worries and compulsions include public toilets, chemicals, shaking hands, door handles, public telephones and surgery. Feelings of mental contamination can be evoked by times when a person perhaps felt badly treated, physically or mentally, through critical or verbally abusive remarks (Greist et al., 1995).

\section{CAUSES}

Actually, the exact cause of obsessive compulsory disorder is not known. While researchers have not yet identified any gene that causes OCD; there is some evidence that genetics may affect who develops the disorder. Children who have family members with OCD have a greater chance of developing OCD early in life. Medical researchers believe that in this case an autoimmune mechanism is to blame. In these cases, treatment with antibiotics may be effective for OCD symptoms. Researchers know that OCD is triggered by communication problems between the brain's deeper structures and the front part of the brain. These parts of the brain primarily use serotonin to communicate. Researchers know that low levels of serotonin can cause OCD symptoms; there is no laboratory test to diagnose OCD (Barr et al., 1992). Serotonin is involved in regulating anxiety .abnormality in the neurotransmitter serotonin, in order to send chemicals messages serotonin must bind to receptor sites located on the neighbouring nerve cells OCD suffers may have blocked or damaged receptor sites preventing serotonin from functioning to full potential .some peoples suffering from mutation in the human serotonin transporter gene.

The three Main Theories that causes Obsessive Compulsive Disorder [OCD]

\section{Biological Theories}

Biological causes of OCD focus on a circuit in the brain which regulates primitive aspects of your behaviour such as aggression, sexuality, and bodily excretions. This circuit relays information from a part of your brain called the orbit frontal cortex to another area called the thalamus and includes other regions such as the caudate nucleus of the basal ganglia. For example, after using the restroom, you may begin to wash your hands to remove any harmful germs you may have encountered (Robertson Pato et al., 992). Since many people with OCD respond to treatment with medications including selective serotonin reuptake inhibitors (SSRIs), which boost the neurochemical serotonin, it has been suggested that dysregulation of this brain circuit may be related to a problem with the serotonin system.

\section{Cognitive-Behavioural Theories}

Almost everyone experiences bizarre or unexpected thoughts throughout the day. According to cognitive-behavioural theories of OCD, if you are vulnerable to OCD you are unable to ignore these thoughts (Baxter et al., 1995). For example, you might believe that having these thoughts means you are going crazy or that you might actually carry out the imagined or feared behaviour (such as stabbing your partner).

\section{Psychodynamic Theories}

Psychodynamic theories of OCD stress that obsessions and compulsions are signs of unconscious conflict that you might be trying to suppress, resolve, or cope with (McDougle et al., 1994). And others may be personal experience, life changes etc.

\section{SYMPTOMS}

The most common types of symptoms (Ciba Geigy, 1998) are

\section{Cleanliness}

They repeatedly wash their hands and clean the house.

\section{Safety}

They are constantly checking whether the doors and windows are secure, whether the stove has been turned off, and so on.

Order

Anxiety that can be seen rearranging books, cutlery, or aligning carpets, pillows and cushions, repeatedly.

\section{Hoarding}

People who find it impossible to dispose of anything. They collect old newspapers, 
clothes, mails, and other objects for no apparent reason.

\section{Counting}

Such people repeatedly count their belongings and other objects used in daily life, such as the number of steps on a staircase etc. If you observe such behavior then that person is suffering from mental health disorder known as OCD (Pfizer, 2000).

\section{PATHOPHYSIOLOGY}

The brain regions impaired in OCD includes Dorso Lateral Prefrontal Cortex (DLPC), Anterior Cingulate Cortex (ACC), Basal Ganglia, Orbito-Frontal Cortex (OFC) etc.

\section{Dorso Lateral Prefrontal Cortex (DLPC)}

It is the most important cortex part for cognitive functions in human beings. The involvement of the DLPC in working memory was initially demonstrated in primate studies. Functional neuroimaging data have shown diminished activity in the DLPC of patients suffering with psychiatric disorders such as major depression and OCD, which may account for the difficulty in overcoming compulsive behaviours (Koponen et al., 1997).

\section{Anterior cingulate cortex (ACC)}

Neuroimaging studies indicated that the ACC is involved in a variety of cognitive processes such as attention, motivation, reward, error detection, working memory, problem solving and action-plan. Excessive activation of ACC has been reported in patients presenting psychiatric disturbances such as phobias, OCD and mood disorders. Moreover, electrophysiological studies in man have demonstrated its particular role in error detection processes (Montgomery et al., 2000).

\section{Basal ganglia}

Basically, the role of the basal ganglia is to integrate the various inputs arriving from the cortex and to use this information for selecting certain motor and/or cognitive programs. The pathological activation of segregated closed loop circuits involving cortex-basal gangliathalamus-cortex pathways would produce reverberating activity and result in a persistent discharge of the innate programs characteristic of OCD.

\section{Orbito-frontal cortex (OFC)}

The OFC is a large brain region, which encompasses both rostral and ventromedial areas. Because, it receives multimodal inputs from the temporal association cortex, amygdala and hypothalamus as well as limbic components of the basal ganglia, it has been viewed as the highest integration centre for emotional processing .The under activity of these regions could probably explain the spatial memory deficits and visual memory deficits observed in OCD patients. The repetitive rituals (compulsions) and aggressive behaviour, which is predominant in OCD patients is probably due to serotonin depletion (Diaz et al., 1997).

\section{DIAGNOSIS}

Steps to help diagnose OCD may include:

\section{Physical exam}

This may be done to help rule out other problems that could be causing your symptoms and to check for any related complications.

\section{Lab tests}

These may include, for example, a complete blood count (CBC), a check of your thyroid function, and screening for alcohol and drugs (Gold stein, 1995).

\section{Psychological evaluation}

This includes discussing your thoughts, feelings, symptoms and behaviour patterns. With your permission, this may include talking to your family or friends.

\section{Diagnostic criteria for OCD}

Your doctor may use criteria in the Diagnostic and Statistical Manual of Mental Disorders (DSM-5), published by the American Psychiatric Association.

\section{TREATMENT}

Obsessive-compulsive disorder treatment may not result in a cure, but it can help bring symptoms under control so that they don't rule your daily life. Some people need treatment for the rest of their lives. You may be often talking about therapies for OCD, either on their own, or along with medication (Pastuszak, 1993). The two main treatments for OCD are psychotherapy and medications. Often, treatment is most effective with a combination of these.

\section{Psychotherapy}

1. Cognitive behavioural therapy (CBT)

A type of psychotherapy, is effective for many people with OCD. Exposure and response prevention (ERP), a type of CBT therapy, involves gradually exposing you to a feared object or obsession, such as dirt, and having you learn healthy ways to cope with your anxiety. ERP takes effort and practice, but you 
may enjoy a better quality of life once you learn to manage your obsessions and compulsions.

Therapy may take place in individual, family or group sessions (Burch et al., 1992).

\section{Exposure and response prevention (ERP)}

Exposure and response prevention (ERP) is specifically designed for OCD. It encourages you to confront your obsessions and resist the urge to carry out compulsions. Instead of performing your usual compulsion, you will be encouraged to try and tolerate the anxiety.ERP helps you to see that the uncomfortable feelings will eventually go away even if you don't perform a compulsion. This type of therapy can be challenging and may make you feel more anxious at first. It's a good idea to talk to a health care professional before you start about what it involves, and whether you are in a good place to start it (Taddio et al., 1966).

\section{Medications}

Certain psychiatric medications can help control the obsessions and compulsions of OCD. Most commonly, antidepressants are tried first. Antidepressants approved by the Food and Drug Administration (FDA) to treat OCD include Clomipramine (Anafranil) for adults and children 10 years and older, Fluoxetine (Prozac) for adults and children 7 years and older, Fluvoxamine for adults and children 8 years and older, Paroxetine (Paxil, Pexeva) for adults only, Sertraline (Zoloft) for adults and children 6 years and older. However, your doctor may prescribe other antidepressants and psychiatric medications.

\section{CONCLUSION}

OCD is an anxiety disorder featuring intrusive and troubling thoughts. The Patient affected by OCD feels compelled to carry out certain abnormal behaviours, although he recognizes that his behaviour is at times irrational. Entire brain functioning is disturbed in patients suffering from OCD, thereby producing devastating effects at the work-place as well as at homes of the patients. OCD is a complicated disorder. Selective serotonin reuptake inhibitors (SSRIs) and to some extent antidepressants form the main stay in the treatment of OCD. Most of the OCD cases are incurable but those can be managed by different medication and therapy.

\section{REFERENCES}

1. Lysaker PH, Bryson GJ, Marks KA, Greig TC and Bell MD. Association of obsessions and compulsions in schizophrenia with neuro cognition and negative symptoms. The Journal of Neuropsychiatry and Clinical Neurosciences. 2000;14(4):449-453.

2. Angst I, Gamma A, Endrass I, Hantouche E, Good R, Ajdacic V, Eich $D$ and Rossler W. Obsessive compulsive syndromes and disorders: Significance of comorbidity with bipolar and anxiety syndromes. European Archives of Psychiatry and Clinical Neuroscience. 2005;255(1):65-71 .

3. Ruscio AM, Stein DJ, Chiu WT and Kessler RC. The epidemiology of obsessive-compulsive disorder in the National Comorbidity Survey Replication. Molecular Psychiatry. 2010;15(1):53-63.

4. Kessler RC, Chiu WT and Demler O. Prevalence, severity, and comorbidity of 12-month DSM-IV disorders in the National Comorbidity Survey Replication. Arch Gen Psychiatry. 2005;62(6):617-627.

5. Jenike MA. Clinical practice. Obsessive compulsive disorder. The New England Journal of Medicine. 2004;350(3):259-265.

6. Abramowitz JS, Taylor S and Mckay D. Obsessive-compulsive disorder. Lancet. 2009;374(9688):491-499.

7. Ab Boileau B. A review of obsessive compulsive disorder in children and adolescents. Dialogues in Clinical Neuroscience. 2011;13(4):401-411.

8. Germana Moretti. What every psychiatrist should know about PANDAS: A Review. Clinical Practice and Epidemiology in Mental health. 2011;4(1):13-17.

9. Greist JH, Jefferson JW and Kobhan KA. Efficacy and tolerability of serotonin transport inhibitors in obsessive-compulsive disorder. Archives of General Psychiatry. 1995;52(1):53-60.

10. Barr LC, Goodman WK and Price LH. The serotonin hypothesis of obsessive-compulsive disorder: implications of pharmacologic challenge studies. Journal of Clinical Psychiatry. 2015;53(4):17-28.

11. Robertson MM and Yakeley J. Tourette syndrome and obsessivecompulsive disorder. Neuropsychiatry, Baltimore, Williams and Wilkins. 1996;827-870 .

12. Baxter LR. Neuroimaging Studies of human anxiety disorders: Cutting paths of knowledge through the field of 
neurotic

phenomena.

Psychopharmacology.

Fourth Generation of progress. New York, Raven. 1995;1287-1300.

13. Mc Dougle CJ, Goodman WK and Price LH. Dopamine antagonists in tic related and Psychotic spectrum obsessive compulsive disorder. Journal of Clinical psychiatry. 1994;55(3):24-31.

14. Ciba-Geigy. Anafranil package insert. Summit NJ. 1998.

15. Pfizer. Zoloft package insert. New York. 2000.

16. Koponen H, Lepola $U$ and Leinonen $E$. Citalopram in the treatment of obsessive compulsive disorder: An open pilot study. Acta Psychiatrica Scandinavica. 1997;96(5);343-346.

17. Montgomery S, Kasper S, BangHedegaard $\mathrm{K}$ and Lundbeck $\mathrm{H}$. The SSRI citalopram is effective in the obsessive-compulsive disorder: Results from a double-blind, fixed dose, placebo-controlled trail. Presented at the Annual meeting of the American psychiatric Association, Chicago. 2000;13-18.

18. Diaz SF, Grush LR, Sichel DA and Cohen LS. Obsessive compulsive disorder in pregnancy and the puerperium. American Psychiatric press, Washington.1997;97-112.

19. Gold stein DJ. Effects of third trimester fluoxetine exposure on the new born. Journal of Clinical Psychopharmacoologyl. 1996;15(6):417-420.

20. Pastuszak A, Schick- Poschetto B and Zuber C. Pregnancy outcome following first trimester exposure to fluoxetine (Prozac). JAMA. 1993;269(17):2246-2248.

21. Burch KJ and Wells BG. Fluoxetine/norfluoxetine concentration in human milk. Paediatrics. 1992;89(4):676-677.

22. Taddio A. Excretion of fluoxetine and its metabolite, norfluoxetine, in human breast milk. Journal of Clinical Pharmacology. 1996;36(1):42-47. 